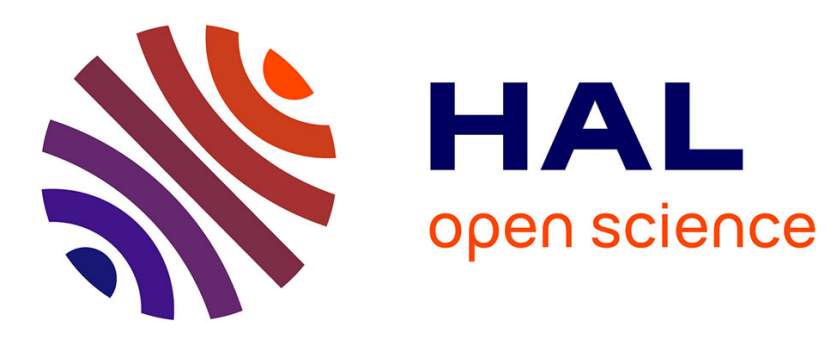

\title{
Influence functions, followers and command games
}

\author{
Michel Grabisch, Agnieszka Rusinowska
}

\section{To cite this version:}

Michel Grabisch, Agnieszka Rusinowska. Influence functions, followers and command games. Games and Economic Behavior, 2011, 72 (1), pp.123-138. 10.1016/j.geb.2010.06.003 . halshs-00583867

\section{HAL Id: halshs-00583867 https://shs.hal.science/halshs-00583867}

Submitted on 6 Apr 2011

HAL is a multi-disciplinary open access archive for the deposit and dissemination of scientific research documents, whether they are published or not. The documents may come from teaching and research institutions in France or abroad, or from public or private research centers.
L'archive ouverte pluridisciplinaire HAL, est destinée au dépôt et à la diffusion de documents scientifiques de niveau recherche, publiés ou non, émanant des établissements d'enseignement et de recherche français ou étrangers, des laboratoires publics ou privés. 


\title{
Influence functions, followers and command games
}

\author{
MICHEL GRABISCH^ and AGNIESZKA RUSINOWSKA \\ Université Paris I Panthéon-Sorbonne \\ Centre d'Economie de la Sorbonne, 106-112 Bd de l'Hôpital, 75013 Paris, France \\ Phone: (+33) 144078 285, Fax: (+33) 144078301 \\ michel.grabisch@univ-paris1.fr \\ agnieszka.rusinowska@univ-paris1.fr
}

\begin{abstract}
We study and compare two frameworks: a model of influence, and command games. In the influence model, in which players are to make a certain acceptance/rejection decision, due to influence of other players, the decision of a player may be different from his inclination. We study a relation between two central concepts of this model: influence function, and follower function. We deliver sufficient and necessary conditions for a function to be a follower function, and we describe the structure of the set of all influence functions that lead to a given follower function. In the command structure introduced by $\mathrm{Hu}$ and Shapley, for each player a simple game called the command game is built. One of the central concepts of this model is the concept of command function. We deliver sufficient and necessary conditions for a function to be a command function, and describe the minimal sets generating a normal command game. We also study the relation between command games and influence functions. A sufficient and necessary condition for the equivalence between an influence function and a normal command game is delivered.
\end{abstract}

\section{JEL Classification: C7, D7}

Keywords: influence function, follower function, lower and upper inverses, kernel, command game, command function, minimal sets generating a command game

\section{Introduction}

Success of every activity in life depends to a high degree on how well one can influence others. The influence ability helps when creating a favorable impression on others about ourselves and our achievements, e.g., when negotiating for grades, having a job interview, implementing ideas in an organization, or making crucial economic and political decisions.

During the last three decades, the political economic literature has offered many theoretical and empirical studies of political influence and power in groups (see e.g. [30] for a short survey). Although many works on noncooperative models have characterized the field of political economy, a cooperative approach to model influence and power in groups has been applied as well and it is still of great interest. Since influence is present both in noncooperative and cooperative environments, it should be studied by using tools of both noncooperative and cooperative game theory. These two approaches are complementary to each other when studying influence, and hence none of them should be ignored, but they should be rather compared to each other. Applying both the noncooperative and cooperative approaches can give a real picture of what is going on in the world based on interaction between people, and can better explain the influence phenomenon. Starting with a lack of cooperation, one might be better off by switching to a cooperative attitude.

\footnotetext{
* Corresponding author
} 
One of the natural phenomena related to influence and interaction among agents is obviously the concept of leadership. According to power and influence theory of leadership, this concept is based on the form of relationships between people rather than on the abilities of a single person. In [4], the set of outcomes sustainable by a leader with the power to make suggestions in games is examined. These suggestions are important even if players can communicate and form coalitions. The author considers both finitehorizon games and infinite-horizon two-player repeated games. In order to understand how power, influence, and leadership are related to various facets of organizational life at the individual, group and macro levels, network formation is usually applied. The literature on network formation has been growing up very fast for the last decade (see, e.g., $[20,19,2])$. Many noncooperative models of network formation have been investigated (see, e.g., [1]), but also a cooperative approach to networks has been applied (see, e.g., [18]). In [21] the authors propose a graphical representation for noncooperative games called multi-agent influence diagrams (MAIDs), which represent decision problems involving multiple agents. One also needs to mention the use of social networks in studying influence, because, as mentioned in [13], individual decisions and strategic interaction are both embedded in a social network. In [24], for instance, the author stresses the fact that decisions of individuals are often influenced by the decisions of other individuals. She considers a network of interacting agents whose actions are determined by the actions of their neighbors, according to a diffusion rule.

Another approach to modeling players' interactions by the use of a social network has been proposed in [12]. In this model, each player is assumed to have an inclination to say either YES or NO which, due to influence by other voters, may be different from the decision of the player. The influence model introduced in [12] is broader than voting models, because in the influence model the analysis begins not in voting itself, but 'one step earlier', that is, in the framework of original inclinations of voters. Influence by other players in this model means that a player's vote (decision) is different from his original inclination. Formally, the influence is expressed by an influence function, which assigns to each inclination vector (i.e., a vector describing the inclinations of all players) a decision vector (i.e., a vector indicating the decisions of the players). The approach based on influence functions is not cooperative in essence, since it does not involve any notion of game in the cooperative sense. Rather, since influence is a dynamical concept which leads to some equilibrium (final decision after several steps of influence), it has a noncooperative flavor. One of the tools that describes the influence function is the concept of a follower of a given coalition under a given influence function, that is, a voter who always follows the inclination of the coalition in question. Formally, a follower function, which assigns to each coalition the set of its followers, is defined. This influence model is studied e.g. in [10] where, in particular, we introduce weighted influence indices, and consider different influence functions.

Since influence among players is one of the natural phenomena that may appear in particular in voting situations, modeling interaction among voters via voting games has been also presented in the literature. Among concepts related to this topic it is worth mentioning the notion of influence relation in simple games, which was introduced fifty years ago in [17], to qualitatively compare the a priori influence of voters in a simple game. As defined in [17], in a simple game, where players can vote either YES or NO, voter $k$ is said to be at least as influential as voter $j$, if whenever $j$ can transform a losing coalition 
into a majority by joining it, voter $k$ can achieve the same ceteris paribus. Very recently, in [29] the influence relation has been extended to voting games with abstention. The concept of interaction among players in a cooperative game is also studied, for instance, in [8], where players in a coalition are said to exhibit a positive (negative) interaction when the worth of the coalition is greater (smaller) than the sum of the individual worths.

Another interesting model related to the topic in question has been recently presented in $[15,16]$, where the command structure of $[28]$ is applied to model players' interaction relations by simple games. This approach is typically of cooperative nature. For each player, boss sets and approval sets are introduced, and based on these sets, a simple game called the command game for a player is built. Given a set of command games, the command function is defined, which assigns to each coalition the set of all players that are 'commandable' by that coalition. In [9] we compare the framework of command games with the influence model. In particular, we define several influence functions which capture the command structure. These functions are compatible with the command games, in the sense that each commandable player for a coalition in the command game is a follower of the coalition under the command influence function. Some of the presented influence functions are equivalent to the command games: An influence function and a command game are said to be equivalent if the follower function of this influence function is identical to the command function in this command game. For some influence functions we define the equivalent command games. Moreover, we show that not for all influence functions the compatible command games exist.

Although both cooperative and noncooperative approaches to power and influence have been presented in the economic literature, research on the relations between these two approaches has not been conducted frequently so far. The exception in the field of voting power can be found, e.g., in [22], where a noncooperative interpretation of power indices has been provided. The authors model noncooperative bargaining processes and show how the power indices (i.e. the Shapley-Shubik index) can be interpreted as measures of bargaining power that appear as limit cases (see also [23] for cooperative bargaining foundation of this index).

Our aim is to model influence in a broad sense, by studying both the noncooperative and cooperative approaches to influence in groups and, in particular, by determining links between these two approaches. An advantage of our influence framework is its generality which allows to cover many different situations. This is due to the influence function which can be defined arbitrarily. On the one hand, our model covers noncooperative aspects, when an individual tries to change the opinion of other agents and makes them decide differently from their preliminary plans in order to end up in the individual's preferable outcome. On the other hand, the model has also cooperative features with individuals who form coalitions and try to 'win the game' by cooperation.

In this paper we continue our work on influence presented in $[9,10]$. While in the two previous papers on influence we focus on concrete examples, for instance, we define several influence functions and study their properties, the aim of the present paper is to establish exact relations between the key concepts of the influence model and the framework of command games. To be more precise, the aims and main results of this paper are the following:

- studying the exact relation between an influence function and a follower function - We deliver sufficient and necessary conditions for a function to be the follower function of 
some influence function. Given a follower function, we find the smallest and greatest influence functions, called the lower and upper inverses, that lead to this follower function. Moreover, we describe the structure of the set of all influence functions that lead to a given follower function. This structure happens to be a distributive lattice, and we indicate how to compute it.

- studying the exact relation between a command game and a command function - We deliver sufficient and necessary conditions for a function to be the command function of some command game. Moreover, we describe the minimal sets (winning coalitions) generating a normal command game.

- studying the exact relation between a command game and an influence function - A sufficient and necessary condition for the equivalence between an influence function and a normal command game is delivered. We calculate the kernel of an influence function equivalent to a normal command game. Several examples that illustrate the concepts studied and results obtained in this paper are presented.

The paper is structured as follows. In Section 2 we present basic notations and definitions, related to partially ordered sets. Section 3 concerns the model of influence. We recapitulate briefly the model, and study the relation between influence functions and followers functions. In Section 4, the relation between a command game and a command function, and the relation between a command game and an influence function, are studied. In Section 5, we give some concluding remarks, enhancing the most important results of the paper. All the notions are illustrated by several examples. Long and technical proofs, as well as the technical material on partially ordered sets and lattices, are put in the appendix.

\section{Some notations and definitions}

We give here some essential definitions and notations used in the paper. We begin by giving some conventions for sets. We often omit braces for sets if no confusion occurs, e.g., $N \backslash\{k\}, S \cup\{k\}$ will be written $N \backslash k, S \cup k$, etc. Set complementation will be often denoted by a bar, i.e., $\bar{S}:=N \backslash S$, where $N$ is the referential set, and $S$ a subset of it.

Given a finite set $N$, we often deal in this paper with functions from $2^{N}$ to $2^{N}$. Similarly as we write for real-valued functions $f \leq g$ for $f(x) \leq g(x)$ for all $x$, we write for two functions $F, G: 2^{N} \rightarrow 2^{N}$ that $F \leq G$ if $F(S) \subseteq G(S)$ for all $S \subseteq N$.

If neither $F \leq G$ nor $G \leq F$ hold, $F$ and $G$ are said to be incomparable. As usual, $F=G$ means $F(S)=G(S)$ for all $S \subseteq N$.

$F: 2^{N} \rightarrow 2^{N}$ is isotone or monotone nondecreasing if $S \subseteq T \subseteq N$ implies $F(S) \subseteq$ $F(T)$. If the first inclusion is reversed, then $F$ is said to be antitone or monotone nonincreasing. A function is monotone if it is either isotone or antitone.

A partially ordered set $(P, \leq)$ or poset for short, is a set $P$ endowed with a partial order $\leq$, that is, a binary relation being reflexive, antisymmetric and transitive ${ }^{1}$. A lattice $L$ is a poset such that for any $x, y \in L$ their least upper bound, denoted by $x \vee y$, and greatest lower bound $x \wedge y$ always exist. $\left(2^{N}, \subseteq\right)$ is an example of lattice (called Boolean), as well as the set of functions from $2^{N} \rightarrow 2^{N}$, endowed with the above defined order.

\footnotetext{
${ }^{1}$ We give here and after only the very minimum material for the understanding of the paper. We recommend [3] for more details on posets and lattices.
} 
Generalizing the notion of interval, for two elements $x, y \in P$ such that $x \leq y$, we write $[x, y]:=\{z \mid x \leq z \leq y\}$. Also we write $] x, y]$ if $x$ is excluded from the interval, similarly for $[x, y[$. This notation will be often used in the sequel, for subsets and functions.

Given $x \in P$, a predecessor of $x$ is any element $y$ such that $y \leq x$.

\section{Influence functions and follower functions}

\subsection{The model of influence}

The framework of influence that we study in this paper has been originally introduced in [12], and next analyzed e.g. in [9-11,25-27]. We consider a social network with the set of players (agents, voters) denoted by $N:=\{1, \ldots, n\}$. The players have to make a certain acceptance/rejection decision. Each player has an inclination either to say YES (denoted by +1 ) or NO (denoted by -1$)$. An inclination vector $i=\left(i_{1}, \ldots, i_{n}\right)$ is an $n$ vector consisting of ones and minus ones, and indicating the inclinations of all players. Let $I:=\{-1,+1\}^{n}$ be the set of all inclination vectors, and for any coalition $S \subseteq N$, $|S| \geq 1$, let $I_{S}$ denote the set of all inclination vectors under which all members of $S$ have the same inclination, i.e.,

$$
I_{S}:=\left\{i \in I \mid \forall k, j \in S, i_{k}=i_{j}\right\} .
$$

For convenience, we denote $(1,1, \ldots, 1) \in I$ by $1_{N}$, similarly for $-1_{N}$, and also for mixed cases like $\left(1_{S},-1_{N \backslash S}\right)$. This last notation suggests to use the more compact notation $S$, i.e., the set of YES voters, to denote the inclination vector $\left(1_{S},-1_{N \backslash S}\right)$. This set notation will be used most often in the paper.

It is assumed that players may influence each other, and due to the influences in the network, the (final) decision of a player may be different from his (original) inclination. In other words, each inclination vector $i \in I$ is transformed into a decision vector $B i$, where $B: I \rightarrow I, i \mapsto B i$ is the influence function. The decision vector $B i=\left((B i)_{1}, \ldots,(B i)_{n}\right)$ is an $n$-vector consisting of ones and minus ones, and indicating the decisions made by all players. The set of all influence functions is denoted by $\mathcal{B}$. Using the set notation, if $i$ corresponds to $S$, we denote $B i$ by $B(S)$, and $B(S) \subseteq N$ is the set of voters whose (final) decision is YES. Hence, an influence function can be seen as a mapping from $2^{N}$ to $2^{N}$.

One of the main concepts of the influence model is the concept of a follower of a given coalition, that is, a voter who 'always' follows the inclination of the coalition in question. 'Always' means here in all cases in which all members of the coalition have the same inclination. Let $B \in \mathcal{B}$. The follower function of $B$ is a mapping $F_{B}: 2^{N} \rightarrow 2^{N}$ defined as

$$
F_{B}(S):=\left\{k \in N \mid \forall i \in I_{S},(B i)_{k}=i_{S}\right\}, \quad \forall S \subseteq N, S \neq \emptyset,
$$

and $F_{B}(\emptyset):=\emptyset . F_{B}(S)$ is the set of followers of $S$ under $B$. In [10] it is shown that $F_{B}$ is isotone, and $F_{B}(S) \cap F_{B}(T)=\emptyset$ whenever $S \cap T=\emptyset$. The set of all follower functions is denoted by $\mathcal{F}$. In set notation, the definition of the follower function becomes:

$$
F_{B}(S)=\bigcap_{S^{\prime} \supseteq S} B\left(S^{\prime}\right) \cap \bigcap_{S^{\prime} \subseteq N \backslash S} \overline{B\left(S^{\prime}\right)}, \quad \forall S \subseteq N, S \neq \emptyset,
$$

and $F_{B}(\emptyset):=\emptyset$, as it can be checked. 
Assume $F_{B}$ is not identically the empty set. The kernel of $B$ is the following collection of sets:

$$
\mathcal{K}(B):=\left\{S \in 2^{N} \mid F_{B}(S) \neq \emptyset \text {, and } S^{\prime} \subset S \Rightarrow F_{B}\left(S^{\prime}\right)=\emptyset\right\} .
$$

The kernel is well defined due to isotonicity of $F_{B}$. It is the set of minimal coalitions having followers, or put otherwise, the set of 'truly' influential coalitions.

Example 1. We apply the basic influence concepts to the Confucian model of society mentioned in [16], and later analyzed in [9]. A four-member society is considered, i.e., $N=\{1,2,3,4\}$, with the king (1), the man (2), the wife (3), and the child (4). Hence, the set of all inclination vectors is $I=\{-1,+1\}^{4},|I|=16$. The principles which the society should follow in a decision-making process are the following:

(i) The man follows the king;

(ii) The wife and the child follow the man;

(iii) The king should respect his people.

The society with the rules mentioned above can be modeled as a 4-player social network in which some players are influenced (or follow) the other players. The principles (i) and (ii) are rather straightforward to define in terms of an influence function: a decision of player 2 always coincides with the inclination of player 1, while players 3 and 4 always decide according to the inclination of player 2. However, the rule (iii) can be interpreted in different ways, depending, e.g., on features of the society, like its politics, culture, tradition, history, etc. How can the king interpret the rule (iii) in a decision-making process? Let us consider three different interpretations, denoted by (A), (B), and (C). Figure 1 shows social networks for these three cases. An arc from player $j$ to $k$ means that $j$ influences player $k$, i.e., there is at least one inclination vector under which player $k$ decides according to the inclination of $j$, although these two players in question were inclined differently from each other.
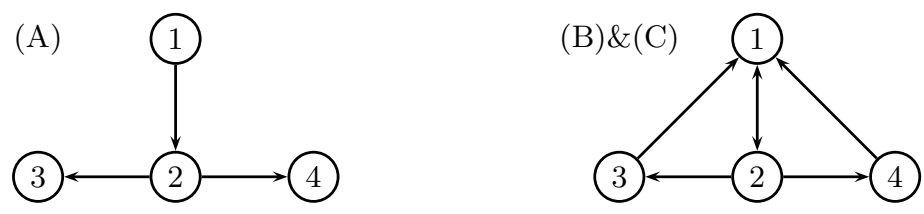

Fig. 1. Social networks for the Confucian model

(A) King follows only himself - In such a society, the king always decides according to his own inclination. Figure 1(A) illustrates this case: the king is never influenced by his people (no arcs going to player 1), but the man is influenced by the king (an arc from player 1 to player 2), and the man influences both his wife and his child (arcs going from player 2 to players 3 and 4). Such a network also models a situation in which the king simply ignores the rule (iii).

(B) King follows only unanimous people - The rule (iii) means for the king to follow his people only if they are all unanimous, otherwise the king decides according to his own inclination. In this case, the king does not follow himself only in two cases when his three people have an inclination different from his own inclination. Figure 1(B) shows this case: there exists a certain influence of the people on the king (there are arcs going from players 2,3 , and 4 to player 1 ), although this influence is quite limited. 
(C) King follows the majority of his people - In such a society, the king follows the majority of his people, i.e., he decides according to the inclination of at least two players from the set $N \backslash\{1\}$. Figure 1(C) illustrates this society. Although the network is the same as in the previous case, the influence function is defined differently from the case (B).

Let the influence functions of the cases (A), (B), and (C) be denoted by $B, B^{\prime}$, and $B^{\prime \prime}$, respectively. The following table presents these influence functions, both in the vector and set notations.

\begin{tabular}{|c|c||c|c||c|c||c|c|}
\hline$i \in I$ & $S \subseteq N$ & $B i$ & $B(S)$ & $B^{\prime} i$ & $B^{\prime}(S)$ & $B^{\prime \prime} i$ & $B^{\prime \prime}(S)$ \\
\hline \hline$(1,1,1,1)$ & $N$ & $(1,1,1,1)$ & $N$ & $(1,1,1,1)$ & $N$ & $(1,1,1,1)$ & $N$ \\
$(1,1,1,-1)$ & 123 & $(1,1,1,1)$ & $N$ & $(1,1,1,1)$ & $N$ & $(1,1,1,1)$ & $N$ \\
$(1,1,-1,1)$ & 124 & $(1,1,1,1)$ & $N$ & $(1,1,1,1)$ & $N$ & $(1,1,1,1)$ & $N$ \\
$(1,-1,1,1)$ & 134 & $(1,1,-1,-1)$ & 12 & $(1,1,-1,-1)$ & 12 & $(1,1,-1,-1)$ & 12 \\
$(-1,1,1,1)$ & 234 & $(-1,-1,1,1)$ & 34 & $(1,-1,1,1)$ & 134 & $(1,-1,1,1)$ & 134 \\
$(1,-1,1,-1)$ & 13 & $(1,1,-1,-1)$ & 12 & $(1,1,-1,-1)$ & 12 & $(-1,1,-1,-1)$ & 2 \\
$(1,1,-1,-1)$ & 12 & $(1,1,1,1)$ & $N$ & $(1,1,1,1)$ & $N$ & $(-1,1,1,1)$ & 234 \\
$(-1,1,1,-1)$ & 23 & $(-1,-1,1,1)$ & 34 & $(-1,-1,1,1)$ & 34 & $(1,-1,1,1)$ & 134 \\
$(1,-1,-1,1)$ & 14 & $(1,1,-1,-1)$ & 12 & $(1,1,-1,-1)$ & 12 & $(-1,1,-1,-1)$ & 2 \\
$(-1,-1,1,1)$ & 34 & $(-1,-1,-1,-1)$ & $\emptyset$ & $(-1,-1,-1,-1)$ & $\emptyset$ & $(1,-1,-1,-1)$ & 1 \\
$(-1,1,-1,1)$ & 24 & $(-1,-1,1,1)$ & 34 & $(-1,-1,1,1)$ & 34 & $(1,-1,1,1)$ & 134 \\
$(1,-1,-1,-1)$ & 1 & $(1,1,-1,-1)$ & 12 & $(-1,1,-1,-1)$ & 2 & $(-1,1,-1,-1)$ & 2 \\
$(-1,1,-1,-1)$ & 2 & $(-1,-1,1,1)$ & 34 & $(-1,-1,1,1)$ & 34 & $(-1,-1,1,1)$ & 34 \\
$(-1,-1,1,-1)$ & 3 & $(-1,-1,-1,-1)$ & $\emptyset$ & $(-1,-1,-1,-1)$ & $\emptyset$ & $(-1,-1,-1,-1)$ & $\emptyset$ \\
$(-1,-1,-1,1)$ & 4 & $(-1,-1,-1,-1)$ & $\emptyset$ & $(-1,-1,-1,-1)$ & $\emptyset$ & $(-1,-1,-1,-1)$ & $\emptyset$ \\
$(-1,-1,-1,-1)$ & $\emptyset$ & $(-1,-1,-1,-1)$ & $\emptyset$ & $(-1,-1,-1,-1)$ & $\emptyset$ & $(-1,-1,-1,-1)$ & $\emptyset$ \\
\hline
\end{tabular}

The sets of followers of each coalition under the three influence functions are as follows:

\begin{tabular}{|r|cccccccc|}
\hline$S$ & $\emptyset$ & 1 & 2 & 3 & 4 & 12 & 13 & 14 \\
\hline$F_{B}(S)$ & $\emptyset$ & 12 & 34 & $\emptyset$ & $\emptyset$ & $N$ & 12 & 12 \\
$F_{B^{\prime}}(S)$ & $\emptyset$ & 2 & 34 & $\emptyset$ & $\emptyset$ & $N$ & 12 & 12 \\
$F_{B^{\prime \prime}}(S)$ & $\emptyset$ & 2 & 34 & $\emptyset$ & $\emptyset$ & 234 & 2 & 2 \\
\hline \hline$S$ & 23 & 24 & 34 & 123 & 124 & 134 & 234 & $N$ \\
\hline$F_{B}(S)$ & 34 & 34 & $\emptyset$ & $N$ & $N$ & 12 & 34 & $N$ \\
$F_{B^{\prime}}(S)$ & 34 & 34 & $\emptyset$ & $N$ & $N$ & 12 & 134 & $N$ \\
$F_{B^{\prime \prime}}(S)$ & 134 & 134 & 1 & $N$ & $N$ & 12 & 134 & $N$ \\
\hline
\end{tabular}

We can see from this table that the follower functions $F_{B}, F_{B}^{\prime}$ and $F_{B}^{\prime \prime}$ are indeed isotone, and for any two disjoint coalitions, the sets of followers of these coalitions under $B, B^{\prime}$ and $B^{\prime \prime}$ are also disjoint. Moreover, the kernel of each influence function is equal to $\mathcal{K}(B)=\mathcal{K}\left(B^{\prime}\right)=\mathcal{K}\left(B^{\prime \prime}\right)=\{\{1\},\{2\}\}$.

\section{$3.2 \quad$ The mapping $\Phi$}

We want to establish the exact relation between two key concepts of the influence model: the influence function, and the follower function. We have seen that an influence function $B$ can be considered as a mapping from $2^{N}$ to $2^{N}$, exactly like follower functions. The cardinality of the set of such mappings is $\left(2^{n}\right)^{\left(2^{n}\right)}=2^{n 2^{n}}$, and there are potentially as many influence functions as follower functions. However, while there is no restriction on $B$, 
$F_{B}$ should satisfy some conditions, like isotonicity. Hence, there are functions in $\left(2^{N}\right)^{\left(2^{N}\right)}$ which cannot be the follower function of some influence function, and consequently, several $B$ 's may have the same follower function. Put differently, we loose some information by considering only $F_{B}$. Formally, this means that the mapping $\Phi: \mathcal{B} \rightarrow\left(2^{N}\right)^{\left(2^{N}\right)}$ defined by

$$
B \mapsto \Phi(B):=F_{B}
$$

is neither a surjection nor an injection. We have $\Phi(\mathcal{B})=: \mathcal{F}$.

The following natural questions may be raised:

(1) Given a function $F: 2^{N} \rightarrow 2^{N}$, which are the sufficient and necessary conditions so that there exists $B \in \mathcal{B}$ such that $F$ is the follower function of $B$, i.e., $F=F_{B}$ ?

(2) If $F: 2^{N} \rightarrow 2^{N}$ is indeed a follower function, can we easily find examples of $B$ 's such that $F_{B}=F$ ?

(3) Moreover, can we find $\Phi^{-1}(F)$, i.e., the set of all influence functions that lead to the follower function $F$ ? What is the (algebraic) structure of $\Phi^{-1}(F)$ ?

The results shown in this subsection answers the first and second questions, and Subsection 3.3 totally solves the third question.

Proposition 1. A function $F: 2^{N} \rightarrow 2^{N}$ is a follower function of some $B \in \mathcal{B}$ (i.e., $F_{B}=F$, or $\Phi(B)=F$ ) if and only if it satisfies the following three conditions:

(i) $F(\emptyset)=\emptyset$;

(ii) $F$ is isotone;

(iii) If $S \cap T=\emptyset$, then $F(S) \cap F(T)=\emptyset$.

Moreover, the smallest and greatest influence functions belonging to $\Phi^{-1}(F)$ are respectively the influence functions $\underline{B}_{F}$ and $\bar{B}_{F}$, defined by, in set notation:

$$
\underline{B}_{F}(S)=F(S), \quad \bar{B}_{F}(S)=\overline{F(\bar{S})}, \quad \forall S \subseteq N .
$$

We call these influence functions the lower and upper inverses of $F$.

(see proof in appendix)

Corollary 1. The function $\Phi$ satisfies the following properties:

(i) For any $B \in \mathcal{B}, \Phi(B) \leq B$.

(ii) The set of fixed points of $\Phi$ (i.e., for which $\Phi(B)=B$ ) is exactly $\mathcal{F}$. Hence, $\Phi^{2}=$ $\Phi^{3}=\cdots=\Phi$.

Proof: (i) Denoting $F:=\Phi(B)$, by definition $B \geq \underline{B}_{F}=F=\Phi(B)$ by (2).

(ii) If $B \in \mathcal{F}$, then $B \in \Phi^{-1}(B)$, hence $\Phi(B)=B$. Conversely, suppose that $B \notin \mathcal{F}$ and $\Phi(B)=B$. But then $B \in \mathcal{F}$, a contradiction. The last affirmation follows from $\Phi(\mathcal{B})=\mathcal{F}$.

Example 2. Consider $F(S)=\emptyset$, for all $S \subseteq N$, which is a follower function. We already know from [10, Prop. 7] that an inverse of $F$ by $\Phi$ is the reversal function -ld, defined by $(-\mathrm{dd}) i:=-i$, for each $i \in I$. Clearly, the lower inverse is the constant function $B \equiv-1_{N}$, while the upper inverse is $B \equiv 1_{N}$. 
Example 3. Consider $F=\mathrm{ld}$, which is a follower function. We know already from [10, Prop. 6] that an inverse of $F$ is the identity function Id. Clearly, the lower and upper inverses collapse to Id. Hence, $\Phi^{-1}(\mathrm{Id})=\{\mathrm{Id}\}$.

Example 4. Let $n=3$ and the following function $F$ be defined as follows:

\begin{tabular}{|r|llllllll|}
\hline$S$ & $\emptyset$ & 1 & 2 & 3 & 12 & 13 & 23 & 123 \\
\hline$F(S)$ & $\emptyset$ & $\emptyset$ & 2 & $\emptyset$ & 2 & 3 & 12 & 123 \\
\hline
\end{tabular}

One can check that it is indeed a follower function. The upper and lower inverses are:

\begin{tabular}{|r|cccccccc|}
\hline$i$ & $\emptyset$ & 1 & 2 & 3 & 12 & 13 & 23 & 123 \\
\hline $\bar{B}_{F} i$ & $\emptyset$ & 3 & 12 & 13 & 123 & 13 & 123 & 123 \\
$\underline{B}_{F} i$ & $\emptyset$ & $\emptyset$ & 2 & $\emptyset$ & 2 & 3 & 12 & 123 \\
\hline
\end{tabular}

\subsection{Structure of $\Phi^{-1}(F)$}

We know that all elements of the inverse of $F$ are between $\underline{B}_{F}$ and $\bar{B}_{F}$, with the usual order $\leq$ on functions. Then $\left(\Phi^{-1}(F), \leq\right)$ is a poset, which is a subset of $\left(\left[\underline{B}_{F}, \bar{B}_{F}\right], \leq\right)$. We write for simplicity

$$
D_{S}:=\bar{B}_{F}(S) \backslash \underline{B}_{F}(S), \quad S \subseteq N .
$$

Hence, an element of $\left[\underline{B}_{F}, \bar{B}_{F}\right]$ is more easily denoted by the $2^{n}$-dim vector $\left(T_{\emptyset}, \ldots, T_{N}\right)$, where $T_{S} \subseteq D_{S}$ for each $S \subseteq N$. With this notation, $\underline{B}_{F}$ and $\bar{B}_{F}$ are denoted by $(\emptyset, \ldots, \emptyset)$ and $\left(D_{\emptyset}, \ldots, D_{N}\right)$ respectively, and $\bar{B}_{F}$ in Example 4 is $(\emptyset, 3,1,13,13,1,3, \emptyset)$.

We begin by a simple but fundamental observation.

Remark 1. Let $F \in \mathcal{F}$. For any $S \subseteq N$, we have $D_{S}=D_{\bar{S}}$. Indeed, since $F(S) \cap F(\bar{S})=\emptyset$ by Proposition 1 (iii),

$$
D_{S}=\bar{B}(S) \backslash \underline{B}(S)=\overline{F(\bar{S})} \backslash F(S)=\overline{F(S)} \backslash F(\bar{S})=\bar{B}(\bar{S}) \backslash \underline{B}(\bar{S})=D_{\bar{S}} .
$$

Due to this, $T_{S}$ and $T_{\bar{S}}$ neither intersect $F(S)$ nor $F(\bar{S})$ (see Figure 2).

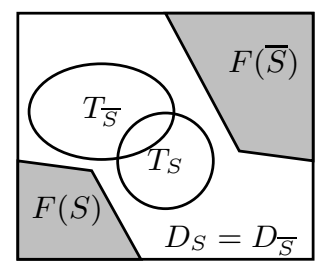

Fig. 2. Set relations between $F(S)$ and $T_{S}$

The next proposition will be useful in the sequel (see proof in Appendix).

Proposition 2. Let $B:=\left(T_{\emptyset}, \ldots, T_{N}\right) \neq \bar{B}_{F}$ be an element of $\Phi^{-1}(F)$. Then for any $S \subseteq N$ such that $D_{S} \backslash T_{S} \neq \emptyset$, and any $k \in D_{S} \backslash T_{S}, B^{\prime}:=\left(T_{\emptyset}, \ldots, T_{S} \cup\{k\}, \ldots, T_{N}\right)$ is an element of $\Phi^{-1}(F)$ if and only if one of the following conditions is not satisfied:

(i) For any $S^{\prime} \supset S, k \in B\left(S^{\prime}\right)$ 
(ii) For any $S^{\prime} \subseteq N \backslash S, k \notin B\left(S^{\prime}\right)$.

We give now the main result of this section, fully describing the structure of $\Phi^{-1}(F)$.

Theorem 1. For any $F \in \mathcal{F}$, the set $\Phi^{-1}(F)$, endowed with the usual ordering of functions, has the following properties:

(i) The greatest and least elements are $\bar{B}_{F}$ and $\underline{B}_{F}=F$.

(ii) It is a lattice, with supremum and infimum given by, for any $S \in 2^{N}$ :

$$
\begin{aligned}
& \left(B \vee B^{\prime}\right)(S):=B(S) \cup B^{\prime}(S) \\
& \left(B \wedge B^{\prime}\right)(S):=B(S) \cap B^{\prime}(S)
\end{aligned}
$$

(iii) $\Phi^{-1}(F)$ is autodual, i.e., $\left(\Phi^{-1}(F), \leq\right)$ and $\left(\Phi^{-1}(F), \geq\right)$ are isomorphic. The duality is expressed as follows: to each element $B:=\left(T_{\emptyset}, \ldots, T_{S}, \ldots, T_{N}\right)$ of $\Phi^{-1}(F)$ corresponds the element $B^{\prime}:=\left(D_{N} \backslash T_{N}, \ldots, D_{\bar{S}} \backslash T_{\bar{S}}, \ldots, D_{\emptyset} \backslash T_{\emptyset}\right)$.

(iv) There are $\sum_{S \subset N}\left|D_{S}\right|$ join-irreducible elements ${ }^{2}$, one for each $k \in D_{S}, S \subseteq N$, either of the form $\left(k_{S} \emptyset\right)$ if this element belongs to $\Phi^{-1}(F)$, otherwise of the form $\left(k_{S} k_{\bar{S}} \emptyset\right)$, where the notation $\left(k_{S} \emptyset\right)$ is a shorthand for $(\emptyset, \ldots, \emptyset, k, \emptyset, \ldots, \emptyset)$, where $k$ is at position $S$, and similarly for $\left(k_{S} k_{\bar{S}} \emptyset\right)$.

(v) The lattice is distributive and its height $t^{3}$ is $h=\sum_{S \subseteq N}\left|D_{S}\right|$.

(see proof in Appendix) We comment about (iv), which is the keypoint of the result. From lattice theory, we know that when the lattice is distributive, it is enough to know its joinirreducible elements, which play a role similar to the basis of a vector space. Specifically, any element of the lattice can be written in a unique way as an (irredundant) supremum of join-irreducible elements. Moreover, we can generate all elements of the lattice as follows. Denote by $\mathcal{J}$ the set of its join-irreducible elements, and endow it with the same order relation $\leq$. Consider an antichain $\mathcal{A}$ of $\mathcal{J}$, that is, a subset of $\mathcal{J}$ such that any two elements $B, B^{\prime}$ of $\mathcal{A}$ are incomparable (i.e., neither $B \leq B^{\prime}$ nor $B^{\prime} \leq B$ hold). Then $B:=\bigvee_{B^{\prime} \in \mathcal{A}} B^{\prime}$ is an element of the lattice $\Phi^{-1}(F)$, and generating the entire lattice amounts to generating all antichains of $\mathcal{J}$.

Concerning (iv) again, checking whether $\left(k_{S} \emptyset\right)$ is an element of $\Phi^{-1}(F)$ is done by using Proposition 2 with $\left(T_{\emptyset}, \ldots, T_{N}\right)=(\emptyset, \ldots, \emptyset)$, i.e., it amounts to check if one of the two following conditions fails:

(i) $\forall S^{\prime} \supset S, k \in F\left(S^{\prime}\right)$

(ii) $\forall S^{\prime} \subseteq N \backslash S, k \notin F\left(S^{\prime}\right)$.

Note that if $\left(k_{S} \emptyset\right)$ is not an element of $\Phi^{-1}(F)$, then necessarily $\left(k_{\bar{S}} \emptyset\right)$ is.

Example 5. (Example 4 continued) Let us compute the join-irreducible elements of $\Phi^{-1}(F)$, with $F$ given in Example 4 . The sets $D_{S}$ are as follows.

\begin{tabular}{|r|cccccccc|}
\hline$S$ & $\emptyset$ & 1 & 2 & 3 & 12 & 13 & 23 & 123 \\
\hline$D_{S}$ & $\emptyset$ & 3 & 1 & 13 & 13 & 1 & 3 & $\emptyset$ \\
\hline
\end{tabular}

\footnotetext{
${ }^{2}$ A join-irreducible element of a lattice covers only one element, where $x$ "covers" $y$ means $x>y$ and there is no $z$ such that $x>z>y$.

${ }^{3}$ A lattice is distributive if $\vee, \wedge$ obey distributivity. The height of a lattice is the length of a longest path from the least to the greatest element. It is a classical result that for a distributive lattice, its height is the number of join-irreducible elements.
} 
The set $\mathcal{J}$ of join-irreducible elements contains 8 elements, given by:

- For $S=1, k=3:\left(3_{1} \emptyset\right)$ belongs to $\Phi^{-1}(F)$, so it is a join-irreducible element.

- For $S=2, k=1$ : $\left(1_{2} \emptyset\right)$ belongs to $\Phi^{-1}(F)$, so it is a join-irreducible element.

- For $S=3, k=1:\left(1_{3} \emptyset\right)$ belongs to $\Phi^{-1}(F)$, so it is a join-irreducible element.

- For $S=3, k=3$ : $\left(3_{3} \emptyset\right)$ belongs to $\Phi^{-1}(F)$, so it is a join-irreducible element.

- For $S=12, k=1$ : $\left(1_{12} \emptyset\right)$ does not belong to $\Phi^{-1}(F)$, so $\left(1_{12} 1_{3} \emptyset\right)$ is a join-irreducible element.

- For $S=12, k=3:\left(3_{12} \emptyset\right)$ does not belong to $\Phi^{-1}(F)$, so $\left(3_{12} 3_{3} \emptyset\right)$ is a join-irreducible element.

- For $S=13, k=1:\left(1_{13} \emptyset\right)$ does not belong to $\Phi^{-1}(F)$, so $\left(1_{13} 1_{2} \emptyset\right)$ is a join-irreducible element.

- For $S=23, k=3:\left(3_{23} \emptyset\right)$ does not belong to $\Phi^{-1}(F)$, so $\left(3_{23} 3_{1} \emptyset\right)$ is a join-irreducible element.

¿From this we can generate all elements of $\Phi^{-1}(F)$. For example, $\left\{\left(1_{12} 1_{3} \emptyset\right),\left(3_{1} \emptyset\right),\left(1_{2} \emptyset\right)\right\}$ is an antichain of $\mathcal{J}$, therefore $\left(3_{1} 1_{2} 1_{3} 1_{12}\right)=(\emptyset, 3,1,1,1, \emptyset, \emptyset, \emptyset)$ is an element of $\Phi^{-1}(F)$.

\section{Command games, command functions, and influence functions}

\subsection{The command games}

We recapitulate briefly the main concepts of the command games introduced by $[15,16]$. Let $N=\{1, \ldots, n\}$ be the set of players (voters). For $k \in N$ and $S \subseteq N \backslash k$ :

- $S$ is a boss set for $k$ if $S$ determines the choice of $k$;

- $S$ is an approval set for $k$ if $k$ can act with an approval of $S$.

It is assumed that no subset can be both a boss set and an approval set, and that any superset (in $N \backslash k$ ) of a boss set (resp. of an approval set) is a boss set (resp. an approval set, provided it is not a boss set). Also, it is assumed that the empty set cannot be a boss set because this does not make sense, but the empty set can be an approval set (which means that player $k$ can act alone). To avoid triviality, it is assumed that both families of boss sets and approval sets cannot be empty.

For each $k \in N$, a simple game $\left(N, \mathcal{W}_{k}\right)$ is built, called the command game for $k$, where the set of winning coalitions is

$$
\mathcal{W}_{k}:=\{S \mid S \text { is a boss set for } k\} \cup\{S \cup k \mid S \text { is a boss or approval set for } k\} \text {. }
$$

Note that due to the above assumptions, this family is never empty and always contains $N$. We call for brevity command game the set $\left\{\left(N, \mathcal{W}_{k}\right), k \in N\right\}$ of command games for each player. We can recover the boss sets for $k$ by

$$
\text { Boss }_{k}=\left\{S \subseteq N \backslash k \mid S \in \mathcal{W}_{k}\right\}=\mathcal{W}_{k} \cap 2^{N \backslash k},
$$

and the approval sets for $k$ by

$$
\operatorname{App}_{k}=\left\{S \subseteq N \backslash k \mid S \cup k \in \mathcal{W}_{k} \text { but } S \notin \mathcal{W}_{k}\right\}
$$


Moreover, we consider the minimal boss sets and the minimal approval sets for $k$

$$
\begin{aligned}
\operatorname{Boss}_{k}^{*}: & =\left\{S \in \operatorname{Boss}_{k} \mid S^{\prime} \subset S \Rightarrow S^{\prime} \notin \operatorname{Boss}_{k}\right\} \\
\operatorname{App}_{k}^{*} & :=\left\{S \in \operatorname{App}_{k} \mid S^{\prime} \subset S \Rightarrow S^{\prime} \notin \operatorname{App}_{k}\right\} .
\end{aligned}
$$

Given a command game $\left\{\left(N, \mathcal{W}_{k}\right), k \in N\right\}$, the command function $\omega: 2^{N} \rightarrow 2^{N}$ is defined as

$$
\omega(S):=\left\{k \in N \mid S \in \mathcal{W}_{k}\right\}, \forall S \subseteq N .
$$

$\omega(S)$ is the set of all members that are 'commandable' by $S$. In [15] it is shown that $\omega(\emptyset)=\emptyset, \omega(N)=N$, and $\omega(S) \subseteq \omega\left(S^{\prime}\right)$ whenever $S \subset S^{\prime}$.

\subsection{Relation between command games and influence functions}

Since the framework of command games and our framework of influence functions address the same problem, a natural question is: what is the relation between the two? We first analyze the former, which will lead us to the natural notion of normal command game.

Let us first give an alternative more compact notation for command games, which will make them closer to influence and follower functions. Indeed, a command game $\left\{\left(N, \mathcal{W}_{k}\right), k \in N\right\}$ can be expressed by its characteristic function $\Omega: N \times 2^{N} \rightarrow\{0,1\}$, with

$$
(k, S) \mapsto \Omega(k, S)= \begin{cases}1, & \text { if } S \in \mathcal{W}_{k} \\ 0, & \text { otherwise. }\end{cases}
$$

The set of such functions is $2^{N \times 2^{N}}$, hence its cardinality is $2^{n 2^{n}}$, which is exactly the cardinality of $\mathcal{B}$. Moreover, command functions are mappings from $2^{N}$ to $2^{N}$, with again the same cardinality $2^{n 2^{n}}$. There exists an obvious bijection between $2^{N \times 2^{N}}$ and $\left(2^{N}\right)^{\left(2^{N}\right)}$, let us call it $\Psi$, defined by

$$
\begin{gathered}
\Psi(\Omega)=\omega, \quad \text { with } \omega(S):=\{k \in N \mid \Omega(k, S)=1\}, \quad \forall S \subseteq N \\
\Psi^{-1}(\omega)=\Omega, \quad \text { with } \Omega(k, S)=1 \text { iff } k \in \omega(S) .
\end{gathered}
$$

Hence, $\omega$ and $\Omega$ are equivalent representations of a command game.

Now, it is obvious from Section 4.1 that any mapping $\Omega$ cannot correspond to a command game, because the definition of boss and approval sets induce some structure on the sets $\mathcal{W}_{k}$. Indeed, if $S^{*}$ is a minimal boss set, then any superset of $S^{*}$ is also a boss set. Denoting by $\uparrow S^{*}$ the set of supersets of $S^{*}$, we have that Boss $_{k}=\bigcup_{S^{*} \in \text { Boss }_{k}^{*}} \uparrow S^{*}$, and the same reasoning can be done for approval sets. In summary, by virtue of (3) we find that

$$
\mathcal{W}_{k}=\uparrow S_{1} \cup \ldots \cup \uparrow S_{l}
$$

where either $S_{j}$ does not contain $k$ ( $S_{j}$ is a minimal boss set for $k$ ), or $S_{j}$ contains $k$ (in this case, $S_{j} \backslash k$ is a minimal approval sets for $k$ ).

An important remark is the following. Suppose that $S_{1}, S_{2}$ in (6) are disjoint. Then it may be the case that $S_{1}$ votes YES and $S_{2}$ votes NO, which would lead to a conflict for $k$. Therefore, it is necessary to impose $S_{1} \cap \cdots \cap S_{l} \neq \emptyset$. Lastly, recall that the empty set cannot be a boss set, hence $\mathcal{W}_{k} \neq \uparrow \emptyset=2^{N}$.

We summarize our findings in the next definition. 
Definition 1. A normal command game $\Omega$ is a set of simple games $\left\{\left(N, \mathcal{W}_{k}\right), k \in N\right\}$ satisfying the two conditions:

(i) For each $k \in N$, there exists a minimal nonempty family of nonempty subsets $S_{1}^{k}, \ldots, S_{l_{k}}^{k}$ (called the generating family of $\mathcal{W}_{k}$ ) such that $\mathcal{W}_{k}=\uparrow S_{1}^{k} \cup \ldots \cup \uparrow S_{l_{k}}^{k}$.

(ii) For each $k \in N, S_{1}^{k} \cap \cdots \cap S_{l_{k}}^{k} \neq \emptyset$.

We denote by $\mathcal{G}$ the set of all normal command games (viewed as a subset of $2^{N \times 2^{N}}$ ). ¿From this definition, we are able to tell whether a given function $\omega$ from $2^{N}$ to $2^{N}$ is indeed a command function of some command game.

Proposition 3. Let $\omega \in\left(2^{N}\right)^{\left(2^{N}\right)}$. Then $\omega$ corresponds to some normal command game, i.e., $\omega \in \Psi(\mathcal{G})$, if and only if the following conditions are satisfied:

(i) $\omega(\emptyset)=\emptyset, \omega(N)=N$;

(ii) $\omega$ is isotone, i.e. it is monotone w.r.t. set inclusion;

(iii) If $S \cap S^{\prime}=\emptyset$, then $\omega(S) \cap \omega\left(S^{\prime}\right)=\emptyset$.

(see proof in Appendix) Observe how close to the characterization of influence functions the result is (see Proposition 1).

Suppose that $\omega \in \Psi(\mathcal{G})$, i.e., it satisfies the conditions of Proposition 3. Then the (unique) corresponding command game $\left\{\left(N, \mathcal{W}_{k}\right), k \in N\right\}$ can be easily determined through its generating families $\left\{S_{1}^{k}, \ldots, S_{l_{k}}^{k}\right\}$ of $\mathcal{W}_{k}$ as follows:

$$
\left\{S_{1}^{k}, \ldots, S_{l_{k}}^{k}\right\}=\left\{S \in 2^{N} \mid \omega(S) \ni k \text { and } S^{\prime} \subset S \Rightarrow \omega\left(S^{\prime}\right) \not \supset k\right\} .
$$

Lastly, for any $\omega \in \Psi(\mathcal{G})$, the notion of kernel of $\omega$, denoted by $\mathcal{K}(\omega)$, is meaningful. It is the collection of minimal coalitions commanding at least one player:

$$
\mathcal{K}(\omega):=\left\{S \in 2^{N} \mid \omega(S) \neq \emptyset, \text { and } S^{\prime} \subset S \Rightarrow \omega\left(S^{\prime}\right)=\emptyset\right\} .
$$

The structure of command games being explicited, we are in position to establish the relation between command games and influence functions. Since command functions and follower functions convey a similar meaning, the following definition is natural.

Definition 2. Let $B$ be an influence function and $\Omega$ be a command game. Then $B$ and $\Omega$ are equivalent if $F_{B}=\omega$.

Due to the previous results, equivalence between influence functions and command games is elucidated, and constitutes the main result of Section 4.

Theorem 2. (i) Let $B$ be an influence function. Then there exists a unique normal command game $\Omega$ equivalent to $B$ if and only if $F_{B}(N)=N$. The generating families $\left\{S_{1}^{k}, \ldots, S_{l_{k}}^{k}\right\}, k \in N$, of $\Omega$ are given by (7), taking $\omega:=F_{B}$. The minimal boss sets and minimal approval sets are:

$$
\operatorname{Boss}_{k}^{*}=\left\{S_{j}^{k} \mid S_{j}^{k} \not \supset k, j=1, \ldots, l_{k}\right\}, \quad \mathrm{App}_{k}^{*}=\left\{S_{j}^{k} \backslash k \mid S_{j}^{k} \ni k, j=1, \ldots, l_{k}\right\} .
$$

(ii) Let $\Omega$ be a normal command game. Then any influence function in $\Phi^{-1}(\omega)$ is equivalent to $\Omega$, in particular the upper inverse $\bar{B}_{\omega}$ and the lower inverse $\underline{B}_{\omega}$. Moreover, the kernel of any influence function $B$ in $\Phi^{-1}(\omega)$ is given by

$$
\mathcal{K}(B)=\min \left(\bigcup_{k \in N}\left\{S_{1}^{k}, \ldots, S_{l_{k}}^{k}\right\}\right)=\mathcal{K}(\omega)
$$

where $\min (. .$.$) means that only minimal sets are selected from the collection.$ 


\subsection{Examples}

In order to illustrate the concepts and results presented in this section, we recall two examples of command games mentioned in [16]. According to Definition 1, both are normal command games.

Example 6. Let us analyze the following command game:

$$
N=\{1,2,3\}, \quad \mathcal{W}_{1}=\{12,13,23,123\}, \quad \mathcal{W}_{2}=\{12,23,123\}, \quad \mathcal{W}_{3}=\{23,123\} .
$$

The command function is:

$$
\begin{gathered}
\omega(1)=\omega(2)=\omega(3)=\emptyset, \quad \omega(12)=\{1,2\}, \quad \omega(13)=\{1\}, \quad \omega(23)=N, \quad \omega(N)=N \\
\mathcal{K}(\omega)=\{12,13,23\} .
\end{gathered}
$$

We can apply (7) and Theorem 2 to this game, which gives

$$
\begin{gathered}
\left\{S_{1}^{1}, \ldots, S_{l_{1}}^{1}\right\}=\{12,13,23\}, \quad\left\{S_{1}^{2}, \ldots, S_{l_{2}}^{2}\right\}=\{12,23\}, \quad\left\{S_{1}^{3}, \ldots, S_{l_{3}}^{3}\right\}=\{23\} \\
\text { Boss }_{1}^{*}=\text { Boss }_{1}=\{23\}, \quad \text { Boss }_{k}^{*}=\text { Boss }_{k}=\emptyset, \text { for } k=2,3
\end{gathered}
$$

$\operatorname{App}_{1}^{*}=\mathrm{App}_{1}=\{2,3\}, \quad \mathrm{App}_{2}^{*}=\{1,3\}, \quad \operatorname{App}_{2}=\{1,3,13\} \quad \mathrm{App}_{3}^{*}=\{2\}, \quad \operatorname{App}_{3}=\{2,12\}$.

It can be checked that the same result is obtained from the families of winning coalitions $\mathcal{W}_{1}, \mathcal{W}_{2}$, and $\mathcal{W}_{3}$. The upper and lower inverses of $\omega$ are

\begin{tabular}{|r|cccccccc|}
\hline$S$ & $\emptyset$ & 1 & 2 & 3 & 12 & 13 & 23 & $N$ \\
\hline $\bar{B}_{\omega}(S)$ & $\emptyset$ & $\emptyset$ & 23 & 3 & $N$ & $N$ & $N$ & $N$ \\
$\underline{B}_{\omega}(S)$ & $\emptyset$ & $\emptyset$ & $\emptyset$ & $\emptyset$ & 12 & 1 & $N$ & $N$ \\
\hline
\end{tabular}

We have, of course, $F_{\underline{B}}=\omega, F_{\bar{B}}=\omega$, and

$$
\mathcal{K}(\underline{B})=\mathcal{K}(\bar{B})=\{12,13,23\}=\min \left(\bigcup_{k \in\{1,2,3\}}\left\{S_{1}^{k}, \ldots, S_{l_{k}}^{k}\right\}\right) .
$$

Example \%. We turn to the Confucian model of society originally mentioned in [15] and presented in Example 1 (see Section 3.1). In [9] we have studied several versions of this model with different $\mathcal{W}_{1}$. We assume now that

$$
\begin{gathered}
N=\{1,2,3,4\}, \quad \mathcal{W}_{1}=\{1234\}, \quad \mathcal{W}_{2}=\{1,12,13,14,123,124,134,1234\} \\
\mathcal{W}_{3}=\mathcal{W}_{4}=\{2,12,23,24,123,124,234,1234\}
\end{gathered}
$$

We have therefore

$$
\begin{gathered}
\omega(1)=\omega(13)=\omega(14)=\omega(134)=\{2\}, \quad \omega(2)=\omega(23)=\omega(24)=\omega(234)=\{3,4\} \\
\omega(3)=\omega(4)=\omega(34)=\emptyset, \quad \omega(12)=\omega(123)=\omega(124)=\{2,3,4\}, \quad \omega(N)=N \\
\mathcal{K}(\omega)=\{\{1\},\{2\}\} .
\end{gathered}
$$


Let us apply (7) to this game. We obtain

$$
\begin{gathered}
\left\{S_{1}^{1}, \ldots, S_{l_{1}}^{1}\right\}=\{1234\}, \quad\left\{S_{1}^{2}, \ldots, S_{l_{2}}^{2}\right\}=\{1\} \\
\left\{S_{1}^{3}, \ldots, S_{l_{3}}^{3}\right\}=\left\{S_{1}^{4}, \ldots, S_{l_{4}}^{4}\right\}=\{2\} .
\end{gathered}
$$

We can also apply Theorem 2 to this game:

$$
\begin{gathered}
\text { Boss }_{1}^{*}=\text { Boss }_{1}=\emptyset, \quad \text { App }_{1}^{*}=\text { App }_{1}=\{234\} \\
\text { Boss }_{2}^{*}=\{1\}, \quad \text { Boss }_{2}=\{1,13,14,134\} \\
\text { Boss }_{3}^{*}=\text { Boss }_{4}^{*}=\{2\}, \quad \text { Boss }_{3}=\{2,12,24,124\}, \quad \text { Boss }_{4}=\{2,12,23,123\} \\
\operatorname{App}_{k}=\text { App }_{k}^{*}=\emptyset \text { for } k=2,3,4 .
\end{gathered}
$$

The upper and lower inverses of $\omega$ are

\begin{tabular}{|r|cccccccc|}
\hline$S$ & $\emptyset$ & 1 & 2 & 3 & 4 & 12 & 13 & 14 \\
\hline $\bar{B}_{\omega}(S)$ & $\emptyset$ & 12 & 134 & 1 & 1 & $N$ & 12 & 12 \\
$\underline{B}_{\omega}(S)$ & $\emptyset$ & 2 & 34 & $\emptyset$ & $\emptyset$ & 234 & 2 & 2 \\
\hline \hline & 23 & 24 & 34 & 123 & 124 & 134 & 234 & $N$ \\
\hline $\bar{B}_{\omega}(S)$ & 134 & 134 & 1 & $N$ & $N$ & 12 & 134 & $N$ \\
$\underline{B}_{\omega}(S)$ & 34 & 34 & $\emptyset$ & 234 & 234 & 2 & 34 & $N$ \\
\hline
\end{tabular}

We have, $F_{\underline{B}}=\omega, F_{\bar{B}}=\omega$, and

$$
\mathcal{K}(\underline{B})=\mathcal{K}(\bar{B})=\{\{1\},\{2\}\}=\min \left(\bigcup_{k \in\{1,2,3,4\}}\left\{S_{1}^{k}, \ldots, S_{l_{k}}^{k}\right\}\right) .
$$

Note that none of the influence functions $B, B^{\prime}$ and $B^{\prime \prime}$ defined in Example 1 is equivalent to this command game with $\mathcal{W}_{1}=\{1234\}$, since $F_{B} \neq \omega, F_{B^{\prime}} \neq \omega$, and $F_{B^{\prime \prime}} \neq \omega$. For instance, $F_{B}(134)=F_{B^{\prime}}(134)=F_{B^{\prime \prime}}(134)=\{1,2\} \neq\{2\}=\omega(134)$.

\section{Summary of results and concluding remarks}

We have tried in this paper to make clear the relationship between two different frameworks for the modeling of influence, namely influence functions and command games. We think useful to emphasize some results and points raised in the paper:

- The notion of equivalence between a command game and an influence function (see Definition 2) is the key notion permitting to compare the two frameworks, and this notion is naturally dictated by the definitions of follower and command functions. Moreover, the notion of equivalence permits also to clarify the operational meaning of command games. Indeed, in the framework of $\mathrm{Hu}$ and Shapley, it is not clearly stated, once the winning coalitions, boss sets and approval sets are fixed, what finally the players will decide in a given voting situation. The link we propose through the follower function permits to know all possible decision vectors from a given inclination vector. Specifically, given a command game $\Omega$, we compute $\omega=\Psi(\Omega)$, then considering $\omega$ as a follower function, we compute $\Phi^{-1}(\omega)$, which is the set of all possible influence functions equivalent to $\Omega$. From a given inclination vector $i$, the set of all possible decision vectors under the command game $\Omega$ is $\left\{B i \mid B \in \Phi^{-1}(\Psi(\Omega))\right\}$. 
- The framework of influence functions is more general than the framework of command games in two aspects. Theorem 2 shows clearly that, firstly, there are influence functions not representable by a normal command game (these are all $B$ 's such that $F_{B}(N) \neq N$ ), and secondly, to each normal command game, it corresponds in general several influence functions which are equivalent to the command game.

- On the other hand, the framework of command games brings an interesting interpretation of the framework of influence functions. The generality of influence functions has to be paid by a relative opacity of its meaning. Given an influence function $B$, it is hard to directly guess what are the influential players, and what is the exact mechanism of influence implemented by $B$. Provided an equivalent command game exists, Theorem 2 (i) brings a nice interpretation of an influence function through (minimal) boss sets and approval sets. One should note also that boss and approval sets are closely linked to the notion of kernel (of an influence function or a command function).

Figure 3 tries to make clear the above points.

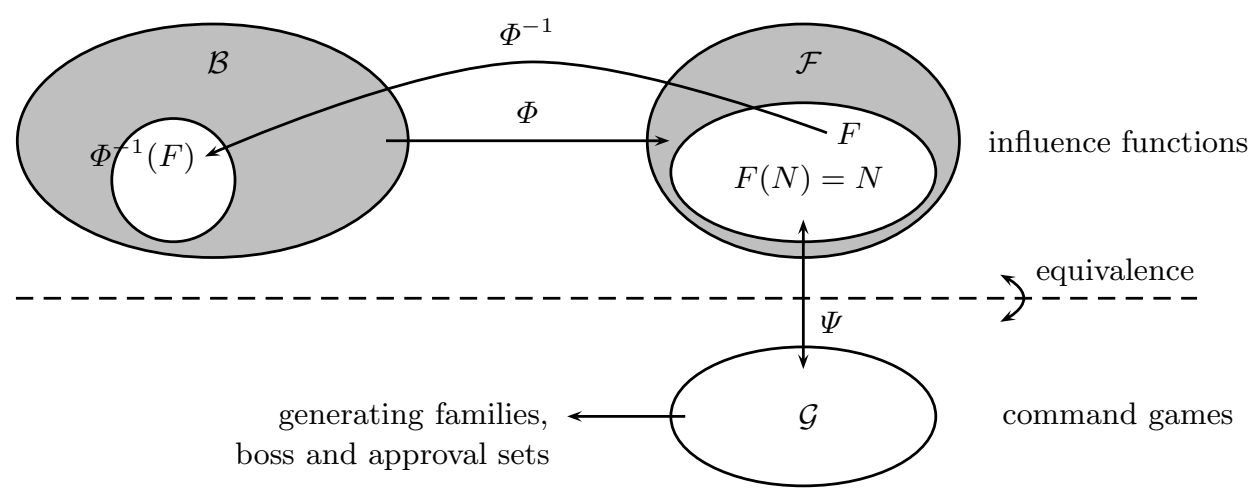

Fig. 3. Relations between influence functions and command games

The results presented in this paper establish a basis on which further research can be undertaken. For instance, a generalized model of influence, in which each player has a continuum of actions, should be investigated. The main aim of this research line is to continue our work on the influence model with an ordered set of possible actions (see [11]). Such an extension of the influence model is related to some works on abstention that have already been presented in the literature on voting (see, e.g. [5-7, 29]), and on multi-choice games [14]. A research agenda concerning our future work on the influence model contains also an introduction of dynamic aspects into the model and a study of the behavior of the series of influence functions.

\section{References}

1. V. Bala and S. Goyal. A noncooperative model of network formation. Econometrica, 68:1181-1229, 2000.

2. F. Bloch and B. Dutta. Communication networks with endogenous link strength. Games and Economic Behavior, 66:39-56, 2009.

3. B. A. Davey and H. A. Priestley. Introduction to Lattices and Orders. Cambridge University Press, 1990. 
4. P. M. DeMarzo. Coalitions, leadership, and social norms: The power of suggestion in games. Games and Economic Behavior, 4:72-100, 1992.

5. D. Felsenthal and M. Machover. Ternary voting games. International Journal of Game Theory, 26:335-351, 1997.

6. D. Felsenthal and M. Machover. The Measurement of Voting Power: Theory and Practice, Problems and Paradoxes. London: Edward Elgar Publishers, 1998.

7. D. Felsenthal and M. Machover. Models and reality: the curious case of the absent abstention. In M. J. Holler and G. Owen, editors, Power Indices and Coalition Formation, pages 87-103. Kluwer, Dordrecht, 2001.

8. M. Grabisch and M. Roubens. An axiomatic approach to the concept of interaction among players in cooperative games. International Journal of Game Theory, 28:547-565, 1999.

9. M. Grabisch and A. Rusinowska. Measuring influence in command games. Social Choice and Welfare, 33:177-209, 2009.

10. M. Grabisch and A. Rusinowska. A model of influence in a social network. Theory and Decision, 2009. Forthcoming.

11. M. Grabisch and A. Rusinowska. A model of influence with an ordered set of possible actions. Theory and Decision, 2010. Forthcoming.

12. C. Hoede and R. Bakker. A theory of decisional power. Journal of Mathematical Sociology, 8:309-322, 1982.

13. D. Hojman and A. Szeidl. Endogenous networks, social games, and evolution. Games and Economic Behavior, 55:112-130, 2006.

14. C. R. Hsiao and T. E. S. Raghavan. Shapley value for multichoice cooperative games. Games and Economic Behavior, 5:240-256, 1993.

15. X. Hu and L. S. Shapley. On authority distributions in organizations: controls. Games and Economic Behavior, 45:153-170, 2003.

16. X. Hu and L. S. Shapley. On authority distributions in organizations: equilibrium. Games and Economic Behavior, 45:132-152, 2003.

17. J. R. Isbell. A class of simple games. Duke Mathematical Journal, 25:423-439, 1958.

18. M. O. Jackson. Allocation rules for network games. Games and Economic Behavior, 51:128-154, 2005.

19. M. O. Jackson and A. Van den Nouweland. Strongly stable networks. Games and Economic Behavior, 51:420-444, 2005.

20. M. O. Jackson and A. Wolinsky. A strategic model of social and economic networks. Journal of Economic Theory, 71:44-74, 1996.

21. D. Koller and B. Milch. Multi-agent influence diagrams for representing and solving games. Games and Economic Behavior, 45:181-221, 2003.

22. A. Laruelle and F. Valenciano. Noncooperative foundations of bargaining power in committees and the Shapley-Shubik index. Games and Economic Behavior, 63:341-353, 2008.

23. A. Laruelle and F. Valenciano. Cooperative bargaining foundations of the Shapley-Shubik index. Games and Economic Behavior, 65:242-255, 2009.

24. D. Lopez-Pintado. Diffusion in complex social networks. Games and Economic Behavior, 62:573-590, 2008.

25. A. Rusinowska. On the not-preference-based Hoede-Bakker index. In L. Petrosjan and V. Mazalov, editors, Game Theory and Applications, volume 13, pages 127-141, New York, 2008. Nova Science Publishers, Inc.

26. A. Rusinowska and H. De Swart. Generalizing and modifying the Hoede-Bakker index. In H. De Swart, E. Orlowska, G. Schmidt, and M. Roubens, editors, Theory and Applications of Relational Structures as Knowledge Instruments II, LNAI 4342, pages 60-88, Heidelberg, 2006. Springer Verlag.

27. A. Rusinowska and H. De Swart. On some properties of the Hoede-Bakker index. Journal of Mathematical Sociology, 31:267-293, 2007.

28. L. S. Shapley. A Boolean model of organization authority based on the theory of simple games. Mimeo, 1994.

29. B. Tchantcho, L. Diffo Lambo, R. Pongou, and B. Mbama Engoulou. Voters' power in voting games with abstention: Influence relation and ordinal equivalence of power theories. Games and Economic Behavior, 64:335-350, 2008.

30. F. van Winden. Interest group behavior and influence. In C. K. Rowley and F. Schneider, editors, The Encyclopedia of Public Choice, pages 118-129. Springer, 2004.

\section{A Proof of Proposition 1}

We already know from [10, Prop. 2] that any follower function fulfills the above three conditions. Take $F: 2^{N} \rightarrow 2^{N}$ satisfying the above conditions. Let us check if indeed $\Phi\left(\underline{B}_{F}\right)=\Phi(F)=: F_{F}$ is equal to $F$. We have to prove that $F_{F}(S)=F(S)$ for every 
$S \subseteq N$. It is true for $S=\emptyset$, by definition of follower functions, and the condition $F(\emptyset)=\emptyset$. Assume $S \neq \emptyset$. Since $F$ is isotone, we have $F\left(S^{\prime}\right) \supseteq F(S)$ for all $S^{\prime} \supseteq S$, which implies $\bigcap_{S^{\prime} \supseteq S} F\left(S^{\prime}\right)=F(S)$. By (iii), $F\left(S^{\prime}\right) \cap F(S)=\emptyset$ for all $S^{\prime} \subseteq N \backslash S$. Therefore, $\bigcap_{S^{\prime} \subseteq N \backslash S} \overline{F\left(S^{\prime}\right)} \supseteq F(S)$. From (1), we deduce then that $F_{F}(S)=F(S)$.

It remains to show that $F$ is the smallest inverse of $F$. Assuming $F \neq \emptyset$, for any $S \subseteq N$ such that $F(S) \neq \emptyset, k \in F(S)$ implies that for any $B \in \Phi^{-1}(F), B(S)$ contains $k$. Therefore $B(S) \supseteq F(S)$ for any $S \subseteq N$. The proof for the upper inverse is analogous.

\section{B Proof of Proposition 2}

We need first a technical lemma.

Lemma 1. Let $B, B^{\prime}$ be identical influence functions except on $S \subseteq N$, where $k \notin B(S)$, and $B^{\prime}(S)=B(S) \cup k$. Assume that $k$ is neither a follower of $S$ in $B^{\prime}$ nor a follower of $\bar{S}$ in $B$ (i.e., $k \notin F_{B^{\prime}}(S), k \notin F_{B}(\bar{S})$ ). Then $F_{B}\left(S^{\prime}\right)=F_{B^{\prime}}\left(S^{\prime}\right)$, for all $S^{\prime} \subseteq N$.

Proof: Since the only change between $B$ and $B^{\prime}$ is the addition of $k$ to $S$, the only change between $F_{B}$ and $F_{B^{\prime}}$ concerns $k$. Hence, we have to consider, for any $S^{\prime} \subseteq N$, the occurrence of two situations: either (i) $k$ is a follower of $S^{\prime}$ for $B$ but no more for $B^{\prime}$, or (ii) $k$ is not a follower of $S^{\prime}$ for $B$ but it becomes for $B^{\prime}$. First observe that the case $S^{\prime}=S$ is done, hence we can discard it from the analysis. Indeed, $k \notin F_{B^{\prime}}(S)$ by hypothesis, and $k \in F_{B}(S)$ is impossible since $k \notin B(S)$. Hence $F_{B}(S)=F_{B^{\prime}}(S)$.

(i) We consider that $k \in F_{B}\left(S^{\prime}\right)$ and $k \notin F_{B^{\prime}}\left(S^{\prime}\right)$. Then for all $S^{\prime \prime} \supseteq S^{\prime}$, we have $k \in B\left(S^{\prime \prime}\right)$, and for all $S^{\prime \prime} \subseteq N \backslash S^{\prime}, k \notin B\left(S^{\prime \prime}\right)$. Suppose that $S^{\prime} \supset S$ or $S$ and $S^{\prime}$ are incomparable with a nonempty intersection. Then $S$ is neither a superset of $S^{\prime}$ nor a subset of $N \backslash S^{\prime}$, which means that $B$ and $B^{\prime}$ are identical on $\left(\uparrow S^{\prime}\right) \cup\left(\downarrow \overline{S^{\prime}}\right)$. Then $k \in F_{B^{\prime}}\left(S^{\prime}\right)$, a contradiction. Suppose that $S^{\prime} \subset S$. Since $S$ is a superset of $S^{\prime}$, by hypothesis we have $k \in B(S)$, which contradicts the definition of $B$.

The remaining case is $S \cap S^{\prime}=\emptyset$. Then any superset of $\bar{S}$ is a superset of $S^{\prime}$, and any subset of $N \backslash \bar{S}=S$ is a subset of $N \backslash S^{\prime}$, which by hypothesis implies that $k \in F_{B}(\bar{S})$. But this contradicts the assumption.

(ii) We consider that $k \notin F_{B}\left(S^{\prime}\right)$ and $k \in F_{B^{\prime}}\left(S^{\prime}\right)$. Then for all $S^{\prime \prime} \supseteq S^{\prime}$, we have $k \in B^{\prime}\left(S^{\prime \prime}\right)$, and for all $S^{\prime \prime} \subseteq N \backslash S^{\prime}, k \notin B^{\prime}\left(S^{\prime \prime}\right)$. Suppose that $S^{\prime} \supset S$ or $S$ and $S^{\prime}$ are incomparable with a nonempty intersection. As said above, $B$ and $B^{\prime}$ are identical on $\left(\uparrow S^{\prime}\right) \cup\left(\downarrow \overline{S^{\prime}}\right)$. Then $k \in F_{B}\left(S^{\prime}\right)$, a contradiction. Suppose that $S^{\prime} \subset S$. Then any superset of $S$ is a superset of $S^{\prime}$, and any subset of $N \backslash S$ is a subset of $N \backslash S^{\prime}$, which by hypothesis implies that $k \in F_{B^{\prime}}(S)$, a contradiction with the assumption.

Finally, suppose that $S \cap S^{\prime}=\emptyset$. Since $S$ is a subset of $N \backslash S^{\prime}$, we have $k \notin B^{\prime}(S)$, which contradicts the definition of $B^{\prime}$.

Proof: (of Proposition 2) Before to show the equivalence, we remark that $k \in D_{S} \backslash T_{S}$ implies that $k \notin F(S)$ and $k \notin F(\bar{S})$ (by definition of $\bar{B}_{F}$, see Figure 2). Remember also that $F=F_{B}$.

Assume that the two conditions hold. Then $k$ is a follower of $S$ for $B^{\prime}$, which implies that $B^{\prime} \notin \Phi^{-1}(F)$. Assume on the contrary that one of the conditions is false. We have to prove that $F_{B^{\prime}}\left(S^{\prime}\right)=F_{B}\left(S^{\prime}\right)$, for all $S^{\prime} \subseteq N$. Consider first the case $S^{\prime}=S$. Since the 
only change concerns $k$, changes of the followers for $B$ and $B^{\prime}$ can only concern $k$. Then we have only one possibility: $k$ is not a follower of $S$ for $B$ but it becomes for $B^{\prime}$. Since one of the conditions fails, $k \in B^{\prime}(S)$ is not sufficient to ensure that $k \in F_{B^{\prime}}(S)$. Hence $k \notin F_{B^{\prime}}(S)$, and since $k \notin F_{B}(\bar{S})$, we are exactly in the conditions of Lemma 1 , which proves the equality of $F_{B}$ and $F_{B^{\prime}}$.

\section{Proof of Theorem 1}

The proof relies on the following fundamental result.

Proposition 4. Let $F \in \mathcal{F}$ be given, and consider $B:=\left(T_{\emptyset}, \ldots, T_{N}\right)$ an element of $\left[\underline{B}_{F}, \bar{B}_{F}\right]$. Then $B \in \Phi^{-1}(F)$ if and only if the following conditions are satisfied:

(i) $\bigcap_{S \ni i} T_{S} \backslash T_{\bar{S}}=\emptyset, \forall i \in N$ (or equivalently $\bigcap_{S^{\prime} \supseteq S} T_{S^{\prime}} \backslash T_{\overline{S^{\prime}}}=\emptyset, \forall S \subseteq N, S \neq \emptyset$ ).

(ii) $\bigcap_{S^{\prime} \in \mathcal{C}} F\left(S^{\prime}\right) \cap \bigcap_{\substack{S^{\prime \prime} \supseteq{ }^{\prime}, S^{\prime \prime} \nsupseteq S^{\prime}, \forall S^{\prime} \in \mathcal{C}}} T_{S^{\prime \prime}} \backslash T_{\overline{S^{\prime \prime}}}=\emptyset$, for any antichain $\mathcal{C}$ in $\left.] S, N\right]$.

Proof: We express $F_{B}(S)$ and see under which conditions the equality $F_{B}(S)=F(S)$ holds for all $S \subseteq N$. Let us remark that the equality always holds for $S=\emptyset$.

Using (1), the definition of $B$, and Figure 2 we have, for any $S \subseteq N, S \neq \emptyset$,

$$
\begin{aligned}
& F_{B}(S)=\bigcap_{S^{\prime} \supseteq S}\left(F\left(S^{\prime}\right) \cup T_{S^{\prime}}\right) \cap \bigcap_{S^{\prime} \subseteq N \backslash S}\left(\overline{F\left(S^{\prime}\right)} \cap \overline{T_{S^{\prime}}}\right) \\
& =\bigcap_{S^{\prime} \supseteq S}\left(F\left(S^{\prime}\right) \cup T_{S^{\prime}}\right) \cap \bigcap_{S^{\prime} \supseteq S}\left(\overline{F\left(\overline{S^{\prime}}\right)} \cap \overline{T_{\overline{S^{\prime}}}}\right) \\
& =\bigcap_{S^{\prime} \supseteq S}\left[\left(F\left(S^{\prime}\right) \cup T_{S^{\prime}}\right) \cap\left(\overline{F\left(\overline{S^{\prime}}\right)} \cap \overline{T_{\overline{S^{\prime}}}}\right)\right]
\end{aligned}
$$

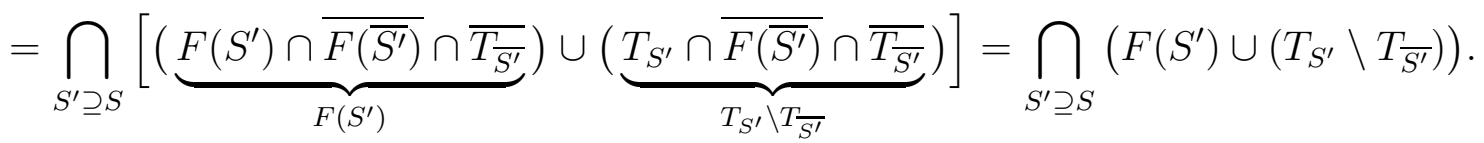

If $S=N$, then we obtain $F_{B}(N)=F(N) \cup\left(T_{N} \backslash T_{\emptyset}\right)$, which implies $T_{N} \backslash T_{\emptyset}=\emptyset$. This is condition (i) for $S=N$. Since condition (ii) is void for $S=N$, we have proved the result for $S=N$. Consider $S \neq \emptyset, N$. Applying distributivity of $\cap$ and $\cup$, we obtain:

$$
F_{B}(S)=\bigcup_{\mathcal{S} \subseteq[S, N]}\left(\bigcap_{S^{\prime} \in \mathcal{S}} F\left(S^{\prime}\right) \cap \bigcap_{S^{\prime} \in[S, N] \backslash \mathcal{S}} T_{S^{\prime}} \backslash T_{\overline{S^{\prime}}}\right) .
$$

Let us process first the simple cases where $\mathcal{S}=\varnothing$ and $\mathcal{S}=[S, N]$. In the first case, we get only the term $\bigcap_{S^{\prime} \supseteq S} T_{S^{\prime}} \backslash T_{\overline{S^{\prime}}}$. In the second case, it remains only $\bigcap_{S^{\prime} \supseteq S} F\left(S^{\prime}\right)$, which is equal to $F(S)$ by isotonicity of $F$.

We suppose now that $\mathcal{S} \neq \varnothing,[S, N]$. Suppose $\mathcal{S} \ni S$. Then by isotonicity of $F$ we get $\bigcap_{S^{\prime} \in \mathcal{S}} F\left(S^{\prime}\right)=F(S)$. Moreover, by Figure 2 and isotonicity of $F$ again, we obtain that $F(S) \cap \bigcap_{S^{\prime} \in[S, N] \backslash \delta} T_{S^{\prime}} \backslash T_{\overline{S^{\prime}}}=\emptyset$.

Suppose on the contrary that $\varnothing \neq \mathcal{S} \in] S, N]$. By isotonicity of $F$, if the family $\mathcal{S}$ has a single minimal element, say $S_{1}$ (i.e., $\mathcal{S} \subseteq\left[S_{1}, N\right]$ and $\left.S_{1} \in \mathcal{S}\right)$, then $\bigcap_{S^{\prime} \in \mathcal{S}} F\left(S^{\prime}\right)=$ 
$F\left(S_{1}\right)$. Similarly, if the family $\mathcal{S}$ has two minimal elements, say $S_{1}, S_{2}$, then $\bigcap_{S^{\prime} \in \mathcal{S}} F\left(S^{\prime}\right)=$ $F\left(S_{1}\right) \cap F\left(S_{2}\right)$, and so on. Let us consider all families $\mathcal{S}$ having as single minimal element $S_{1}$, and consider $\bigcap_{S^{\prime} \in[S, N] \backslash \mathcal{S}} T_{S^{\prime}} \backslash T_{\overline{S^{\prime}}}$. The largest set will be obtained for the smallest family $[S, N] \backslash \mathcal{S}$, hence the largest $\mathcal{S}$, which is $\left[S_{1}, N\right]$. Hence

$$
\bigcup_{\substack{S \subseteq] S, N] \\ \text { minimal element }=S_{1}}}\left(\left(F\left(S_{1}\right) \cap \bigcap_{S^{\prime} \in[S, N] \backslash \mathcal{S}} T_{S^{\prime}} \backslash T_{\overline{S^{\prime}}}\right)=F\left(S_{1}\right) \cap \bigcap_{\substack{S^{\prime} \supseteq S \\ S^{\prime} \grave{\unrhd} S_{1}}} T_{S^{\prime}} \backslash T_{\overline{S^{\prime}}} .\right.
$$

The reasoning easily extends to any number of minimal elements:

$\bigcup_{\substack{S \subseteq] S, N] \\ \text { minimal elements= }=S_{1}, S_{2}}}\left(\left(F\left(S_{1}\right) \cap F\left(S_{2}\right) \cap \bigcap_{S^{\prime} \in[S, N] \backslash \mathcal{S}} T_{S^{\prime}} \backslash T_{\overline{S^{\prime}}}\right)=F\left(S_{1}\right) \cap F\left(S_{2}\right) \cap \bigcap_{\substack{S^{\prime} \\ S^{\prime} \nsupseteq \bar{S}_{1}, S_{2}}} T_{S^{\prime}} \backslash T_{\overline{S^{\prime}}}\right.$

and so on. So we obtain in summary:

$$
F_{B}(S)=\underbrace{F(S)}_{S=[S, N]} \cup(\underbrace{\bigcap_{S^{\prime} \supseteq S} T_{S^{\prime}} \backslash T_{\overline{S^{\prime}}}}_{S=\emptyset}) \cup \bigcup_{\begin{array}{c}
\text { all antichains } \mathcal{E} \text { in }] S, N] \\
\bigcap_{S^{\prime} \in \mathcal{C}} F\left(S^{\prime}\right) \neq F(S)
\end{array}}\left(\bigcap_{S^{\prime} \in \mathcal{C}} F\left(S^{\prime}\right) \cap \bigcap_{\substack{S^{\prime \prime} \supseteq S_{S} \\
S^{\prime \prime} \nsupseteq S^{\prime}, \forall S^{\prime} \in \mathcal{C}}} T_{S^{\prime \prime}} \backslash T_{\overline{S^{\prime \prime}}}\right) .
$$

To get this final expression, observe that the set of all possible configurations of minimal elements of $\mathcal{S}$ coincides with the set of antichains of $] S, N]$. Finally, observe that for any antichain $\mathrm{C}$ in $] S, N]$, we have $\bigcap_{S^{\prime} \in \mathrm{C}} F\left(S^{\prime}\right) \supseteq F(S)$. If equality occurs, then the intersection with any $T_{S^{\prime}} \backslash T_{\overline{S^{\prime}}}, S^{\prime} \in[S, N]$ is empty, by Figure 2 and isotonicity of $F$.

Then clearly, $F_{B}(S)=F(S)$ if and only if each term in parenthesis is the empty set.

Proof: (of Theorem 1) (i) is already known from Prop. 1.

Let us prove first (iii). Take $B:=\left(T_{\emptyset}, \ldots, T_{S}, \ldots, T_{N}\right)$ of $\Phi^{-1}(F)$. Then the two conditions of Prop. 4 hold for all $S \in 2^{N}$. We have to prove that they still hold for $B^{\prime}$, which amounts to replace in these two conditions $T_{S^{\prime}} \backslash T_{\overline{S^{\prime}}}$ by $\left(D \overline{S^{\prime}} \backslash T_{\overline{S^{\prime}}}\right) \backslash\left(D_{S^{\prime}} \backslash T_{S^{\prime}}\right)$.

But since $D_{S^{\prime}}=\overline{F\left(S^{\prime}\right)} \backslash F\left(\overline{S^{\prime}}\right)=D_{\overline{S^{\prime}}}$, and by Figure $2,\left(D_{\overline{S^{\prime}}} \backslash T_{\overline{S^{\prime}}}\right) \backslash\left(D_{S^{\prime}} \backslash T_{S^{\prime}}\right)=T_{S^{\prime}} \backslash T_{\overline{S^{\prime}}}$, which proves the result.

Let us prove (ii). Since $\Phi^{-1}(F)$ is a subset of a product of set lattices, the operations $\vee, \wedge$ defined above are clearly supremum and infimum. We just have to prove that $B \vee B^{\prime}$ and $B \wedge B^{\prime}$ belong to $\Phi^{-1}(F)$ whenever $B, B^{\prime}$ belong to $\Phi^{-1}(F)$, to prove that $\Phi^{-1}$ is a lattice. Due to (iii), we only have to prove it for, e.g., the infimum.

Consider $B:=\left(T_{\emptyset}, \ldots, T_{N}\right)$ and $B^{\prime}=\left(T_{\emptyset}^{\prime}, \ldots, T_{N}^{\prime}\right)$ in $\Phi^{-1}(F)$. Then $B \wedge B^{\prime}=\left(T_{\emptyset} \cap\right.$ $\left.T_{\emptyset}^{\prime}, \ldots, T_{N} \cap T_{N}^{\prime}\right)$. Using Proposition 4 , we have to prove that the two conditions there are satisfied. The first one reads

$$
K:=\bigcap_{S^{\prime} \supseteq S}\left(T_{S^{\prime}} \cap T_{S^{\prime}}^{\prime}\right) \backslash\left(T_{\overline{S^{\prime}}} \cap T_{\overline{S^{\prime}}}^{\prime}\right)=\emptyset, \quad \forall S \subseteq N, S \neq \emptyset .
$$

From the general relation

$$
\left(A \cap A^{\prime}\right) \backslash\left(B \cap B^{\prime}\right)=\left((A \backslash B) \cap A^{\prime}\right) \cup\left(\left(A^{\prime} \backslash B^{\prime}\right) \cap A\right)
$$


we get

$$
K=\bigcap_{S^{\prime} \supseteq \underline{S}}\left[\left(\left(T_{S^{\prime}} \backslash T_{\overline{S^{\prime}}}\right) \cap T_{S^{\prime}}^{\prime}\right) \cup\left(\left(T_{S^{\prime}}^{\prime} \backslash T_{\overline{S^{\prime}}}^{\prime}\right) \cap T_{S^{\prime}}\right)\right] .
$$

Applying distributivity we get

$$
K=\bigcup_{\mathcal{S} \subseteq[S, N]}\left[\bigcap_{S^{\prime} \in \mathcal{S}}\left(T_{S^{\prime}} \backslash T_{\overline{S^{\prime}}}\right) \cap T_{S^{\prime}}^{\prime} \cap \bigcap_{S^{\prime} \in[S, N] \backslash \mathcal{S}}\left(T_{S^{\prime}}^{\prime} \backslash T_{\overline{S^{\prime}}}^{\prime}\right) \cap T_{S^{\prime}}\right]=: \bigcup_{\mathcal{S} \subseteq[S, N]} K_{\mathcal{S}} .
$$

Taking $\mathcal{S}=[S, N]$ or $\varnothing, K_{\mathcal{S}}=\emptyset$, because by Proposition 4 we have $\bigcap_{S^{\prime} \in[S, N]} T_{S^{\prime}} \backslash T_{\overline{S^{\prime}}}=\emptyset$, and the same for $B^{\prime}$. We consider then $K_{\mathcal{S}}$ with $\varnothing \neq \mathcal{S} \subset[S, N]$. Suppose that $K_{\mathcal{S}} \neq \emptyset$, and take any $x \in K_{\mathcal{S}}$. Then we deduce that

(a) $x \in T_{S^{\prime}}$ and $x \in T_{S^{\prime}}^{\prime}$ for all $S^{\prime} \in[S, N]$;

(b) $x \notin T_{\overline{S^{\prime}}}$ for all $S^{\prime} \in \mathcal{S}$, and $x \notin T_{\overline{S^{\prime}}}^{\prime}$ for all $S^{\prime} \in[S, N] \backslash \mathcal{S}$.

From (a), we easily deduce that for all $S^{\prime} \supseteq S$ and all $S^{\prime} \subseteq N \backslash S, x \notin F\left(S^{\prime}\right)$. Indeed, from (a), we know that $x \in D_{S^{\prime}}$, and so $x \notin F\left(S^{\prime}\right)$ for all $S^{\prime} \in[S, N]$. Next, for any $S^{\prime} \subseteq N \backslash S$, we have $\overline{S^{\prime}} \in[S, N]$. Then $x \in D_{\overline{S^{\prime}}}=D_{S^{\prime}}$, which proves again that $x \notin F\left(S^{\prime}\right)$.

¿From this we deduce that in particular $x \notin F(N)$. Let us prove that $x$ necessarily belongs to either $F_{B}(N)$ or $F_{B^{\prime}}(N)$, which causes $F \neq F_{B}$ or $F \neq F_{B^{\prime}}$, a contradiction with the hypothesis. This amounts to prove that $x$ is a follower of $N$ for $B$ or $B^{\prime}$. We know by (a) that $x \in T_{N}$ and $x \in T_{N}^{\prime}$, which proves that $x \in B(N)$ and $B^{\prime}(N)$. By (b), we deduce that $x \notin T_{\emptyset}$ (if $N \in \mathcal{S}$ ) or $x \notin T_{\emptyset}^{\prime}$ (if $N \notin \mathcal{S}$ ). Since $F(\emptyset)=\emptyset$, we deduce that $x \notin B(\emptyset)$ or $x \notin B^{\prime}(\emptyset)$. We turn to the second condition, which reads

$$
K:=\bigcap_{S^{\prime} \in \mathcal{C}} F\left(S^{\prime}\right) \cap \bigcap_{S^{\prime} \in \mathcal{S}_{\mathcal{e}}}\left(T_{S^{\prime}} \cap T_{S^{\prime}}^{\prime}\right) \backslash\left(T_{\overline{S^{\prime}}} \cap T_{\overline{S^{\prime}}}^{\prime}\right)=\emptyset
$$

for all antichain $\mathcal{C} \in], N]$, for all $S \subset N$, and $\mathcal{S}_{\mathcal{C}}:=\left\{S^{\prime \prime} \supseteq S, S^{\prime \prime} \nsupseteq S^{\prime}, \forall S^{\prime} \in \mathcal{C}\right\}$. Proceeding as above, we get after some manipulation

$$
K=\bigcup_{\mathcal{S} \subseteq \mathcal{S}_{\mathcal{C}}}\left[\bigcap_{S^{\prime} \in \mathcal{C}} F\left(S^{\prime}\right) \cap \bigcap_{S^{\prime} \in \mathcal{S}}\left(\left(T_{S^{\prime}} \backslash T_{\overline{S^{\prime}}}\right) \cap T_{S^{\prime}}^{\prime}\right) \cap \bigcap_{S^{\prime} \in \mathcal{S}_{\mathcal{C}} \backslash \mathcal{S}}\left(\left(T_{S^{\prime}}^{\prime} \backslash T \overline{S^{\prime}}\right) \cap T_{S^{\prime}}\right)\right]=: \bigcup_{\mathcal{S} \subseteq \mathcal{S}_{\mathcal{E}}} K_{\mathcal{S}} .
$$

We have to prove that $K_{\mathcal{S}}=\emptyset, \forall \mathcal{S} \subseteq \mathcal{S}_{\mathcal{e}}$. As above, taking $\mathcal{S}=\mathcal{S}_{\mathcal{C}}$ or $\varnothing$ leads to $K_{\mathcal{S}}=\emptyset$ by Proposition 4. We consider then $\varnothing \neq \mathcal{S} \subset \mathcal{S}_{\mathcal{C}}$, and we assume that $K_{\mathcal{S}} \neq \emptyset$, and consider $x \in K_{\mathcal{S}}$. This implies that $x$ belongs to each term of the intersections in $K_{\mathcal{S}}$, hence

(c) $x \in T_{S^{\prime}}$ and $x \in T_{S^{\prime}}^{\prime}$ for all $S^{\prime} \in \mathcal{S}_{\mathcal{C}}$;

(d) $x \notin T_{\overline{S^{\prime}}}$ for all $S^{\prime} \in \mathcal{S}$, and $x \notin T_{\overline{S^{\prime}}}^{\prime}$ for all $S^{\prime} \in \mathcal{S}_{\mathcal{C}} \backslash \mathcal{S}$;

(e) $x \in F\left(S^{\prime}\right), \forall S^{\prime} \in \mathcal{C}$.

Proceeding as above again, we deduce from (c) that

$$
x \notin F\left(S^{\prime}\right) \text { for all } S^{\prime} \in \mathcal{S}_{\mathcal{C}} \text {, and for all } S^{\prime} \subseteq N \backslash S \text { such that } \overline{S^{\prime}} \in \mathcal{S}_{\mathcal{C}} .
$$

Consider $S_{0}$, any maximal element of $\mathcal{S}_{\mathfrak{e}}$. An important fact is to notice that

$$
\forall S^{\prime} \supset S_{0}, \quad \exists S^{\prime \prime} \in \mathcal{C} \text { such that } S^{\prime} \supseteq S^{\prime \prime}
$$


Indeed, $S^{\prime} \supset S_{0}$ implies $S^{\prime} \notin \mathcal{S}_{\mathcal{e}}$. On the other hand, $S^{\prime} \subseteq N$ and $S^{\prime} \supset S_{0} \supseteq S$ implies $S^{\prime} \in[S, N]$. So by definition of $\mathcal{S}_{\mathcal{e}}, S^{\prime} \supseteq S^{\prime \prime}$ for some $S^{\prime \prime} \in \mathcal{C}$.

Let us prove that $x \in F_{B}\left(S_{0}\right)$ or $F_{B^{\prime}}\left(S_{0}\right)$. Since $x \notin F\left(S_{0}\right)$ by (8), this suffices to give a contradiction, hence $K_{\mathcal{S}}=\emptyset$ for all $\mathcal{S}$, which proves that the second condition is fulfilled. For this, we have to prove:

- $\forall S^{\prime} \supseteq S_{0}, B\left(S^{\prime}\right) \ni x$ (or the same with $B^{\prime}$ ). This is true for $S^{\prime}=S_{0}$ by (c). By (9), $S^{\prime} \supseteq S^{\prime \prime}$ for some $S^{\prime \prime} \in \mathcal{C}$. Since $x \in F\left(S^{\prime \prime}\right)$ by assumption, $x \in F\left(S^{\prime}\right)$ too by isotonicity of $F$. Hence, $x \in B\left(S^{\prime}\right)$, and also $x \in B^{\prime}\left(S^{\prime}\right)$.

- $\forall S^{\prime} \subseteq N \backslash S_{0}, B\left(S^{\prime}\right) \not \supset x$ (or the same condition with $B^{\prime}$ ). From (8) we know that $x \notin F\left(N \backslash S_{0}\right)$, hence $x \notin F\left(S^{\prime}\right)$ for all $S^{\prime} \subseteq N \backslash S_{0}$ by isotonicity of $F$. So it remains to show that $x \notin T_{S^{\prime}}$ for all $S^{\prime} \subseteq N \backslash S_{0}$ (or the same with $T_{S^{\prime}}^{\prime}$ ). By (d), we have $x \notin T_{\overline{S_{0}}}$ or $x \notin T_{\overline{S_{0}}}^{\prime}$ (depending whether $S_{0} \in \mathcal{S}$ or not). Let us assume the case $x \notin T_{\overline{S_{0}}}$. By $(\mathrm{e})$, $x \in F\left(S^{\prime \prime}\right)$ for all $S^{\prime \prime} \in \mathcal{C}$, and $F=F_{B}=F_{B^{\prime}}$. This implies that for all $S^{\prime \prime \prime} \subseteq N \backslash S^{\prime \prime}$, $S^{\prime \prime} \in \mathcal{C}, x \notin T_{S^{\prime \prime \prime}}$. It remains to prove that any $S^{\prime} \subset N \backslash S_{0}$ is necessarily a subset of $N \backslash S^{\prime \prime}$ for some $S^{\prime \prime} \in \mathcal{C}$. But this is equivalent to prove that any $S^{\prime} \supset S_{0}$ is a superset of some $S^{\prime \prime} \in \mathcal{C}$, which is exactly (9).

The proof that $B \wedge B^{\prime}$ belongs to $\Phi^{-1}(F)$ is complete. Now, since these infimum and supremum are those of $\prod_{S \subseteq N} 2^{D_{S}}, \Phi^{-1}(F)$ is a sublattice of it.

(iv) and (v). First, since $\Phi^{-1}(F)$ is a sublattice of a distributive lattice, it is distributive. Hence, it is ranked and the length of any maximal chain from bottom to top is the number of join-irreducible elements. Second, the height of the lattice is at most $\sum_{S \subseteq N}\left|D_{S}\right|$ since one adds at least one new element $k$ of some $D_{S}$ at each step, hence this is the maximal number of join-irreducible elements.

We examine now what are the join-irreducible elements. Let us take $S \subseteq N$ such that $D_{S} \neq \emptyset$, and any $k \in D_{S}$. Evidently, $\left(k_{S} \emptyset\right)$ covers only one element, the bottom of the lattice. Hence it is a join-irreducible element provided it belongs to $\Phi^{-1}(F)$. Applying Proposition 2, this amounts to show that at least one of the following conditions is false:

(i) $\forall S^{\prime} \supset S, k \in F\left(S^{\prime}\right)$

(ii) $\forall S^{\prime} \subseteq N \backslash S, k \notin F\left(S^{\prime}\right)$.

If one of the conditions fails, then $\left(k_{S} \emptyset\right)$ is a join-irreducible element. Suppose then that both conditions hold. Then $\left(k_{S} \emptyset\right) \notin \Phi^{-1}(F)$ because $k$ becomes a follower of $S$. It suffices to add $k$ at some position $S^{\prime}$ with $S^{\prime} \subseteq N \backslash S$, to prevent $k$ from becoming a follower of $S$. Observe that $S^{\prime}=N \backslash S$ is always a solution, because $D_{S}=D_{N \backslash S}$, and so $k \in D_{N \backslash S}$. Moreover, it is the only solution, since for any $S^{\prime} \subset N \backslash S, k \notin D_{S^{\prime}}$. Indeed, if $k \in D_{S^{\prime}}$, then $k \in D_{\overline{S^{\prime}}}$, which means that $k \notin F\left(\overline{S^{\prime}}\right)$. But $\overline{S^{\prime}}$ is a proper superset of $S$, so this contradicts assumption (i). Hence $\left(k_{S} k_{\bar{S}} \emptyset\right)$ is an element of $\Phi^{-1}(F)$. Lastly, we have to show that $\left(k_{S} k_{\bar{S}} \emptyset\right)$ covers only one element. Since by assumption $\left(k_{S} \emptyset\right)$ is not an element of $\Phi^{-1}(F)$, it can only cover, either $\left(k_{\bar{S}} \emptyset\right)$ or, if this element does not belong to $\Phi^{-1}(F)$, the bottom element. In both cases we are done, but observe that only the first case can occur. Indeed, by hypothesis, (ii) holds, which makes fail (i) written for $\bar{S}$ instead of $S$.

Since doing so for all $S \subseteq N$ and all $k \in D_{S}$ we have found $\sum_{S \subseteq N}\left|D_{S}\right|$ join-irreducible elements, there cannot be more. 


\section{Proof of Proposition 3}

Suppose $\omega$ corresponds to some normal command game. Then $\omega(\emptyset)=\emptyset$ follows from the fact that $\emptyset \notin \mathcal{W}_{k}, \forall k \in N$. On the other hand, $\omega(N)=N$ since $N \in \mathcal{W}_{k}, \forall k \in N$. Next, take $S \subseteq S^{\prime} \subseteq N$. If $k \in \omega(S)$, then $k \in \omega\left(S^{\prime}\right)$ too due to the definition of $\mathcal{W}_{k}$, which proves that $\omega(S) \subseteq \omega\left(S^{\prime}\right)$. Lastly, if $k \in \omega(S) \cap \omega\left(S^{\prime}\right)$, then both $S, S^{\prime}$ belong to $\mathcal{W}_{k}$, and so they must have a nonempty intersection.

Conversely, assume that $\omega$ fulfills the three conditions, and consider $\Omega=\Psi^{-1}(\omega)$. Since $\omega(N)=N$, each $\mathcal{W}_{k}$ contains $N$, and thus is nonempty. Since $\omega(\emptyset)=\emptyset$, no $\mathcal{W}_{k}$ contains the empty set. Take any $\mathcal{W}_{k}$, and consider $S \in \mathcal{W}_{k}$. Then any $S^{\prime} \supseteq S$ belongs also to $\mathcal{W}_{k}$, since $S \subseteq S^{\prime}$ implies $\omega(S) \subseteq \omega\left(S^{\prime}\right)$. This proves that $\mathcal{W}_{k}$ has necessarily the form $\uparrow S_{1}^{k} \cup \cdots \cup \uparrow S_{l_{k}}^{k}$. It remains to prove that there is no pair of disjoint sets in this family. Assuming $\mathcal{W}_{k}$ contains at least two subsets (otherwise the condition is void), take $S, S^{\prime} \in \mathcal{W}_{k}$ such that $S \cap S^{\prime}=\emptyset$. Then by (iii), $\omega(S) \cap \omega\left(S^{\prime}\right)=\emptyset$, which contradicts that fact that $S, S^{\prime} \in \mathcal{W}_{k}$. 